\title{
GENERAL TREATMENT OF THE SHORT DISTANCE DIVERGENCE IN CLASSICAL TWO-COMPONENT PLASMAS
}

\author{
by R. DEKEYSER
}

Instituut voor theoretische fysica, Arenbergpark, Heverlee (Leuven), België

\section{Synopsis}

It is shown that a set of neighbouring quantum states can be orthogonally transformed into a set of classically behaving localized packets, if their wave functions can be fairly well approximated by the WKB-method and if they lie in a dense part of the energy spectrum. The distribution of the points of localization is the same as predicted by classical statistical mechanics. This is proved in one dimension and for a general spherical potential. It enables one to formulate a partly quantum-mechanical and partly classical description with a smooth transition in between. The short-distance correlation function for oppositely charged particles in a plasma is now easily calculated.

Introduction. In a previous article ${ }^{1}$ ), we made an approximate calculation of the partition function of a hydrogen plasma by combining a classical and a quantum-mechanical description. Such a combined method may be useful for the calculation in a simple way of some other quantities, as e.g. radiation intensities, which would be wrong if calculated classically and very combersome if done quantum-mechanically.

To justify such a procedure, it is necessary that the energy spectrum contains a region where the two descriptions overlap. This means that in this region the quantum states must be very well approximated by their first order expansion in $\hbar$, usually known as the WKB-approximation. Moreover, the density of the energy spectrum must be high enough to permit the construction of wave packets with a narrow spread in energy. The existence of such a region, called the quasi-classical region, depends on the external plasma conditions, which were discussed elsewhere $\left.{ }^{1}\right)$.

The fundamental question, with which we will be concerned here, is to prove that the existence of such a quasi-classical region produces not only the classical behaviour of a wave-packet, but the complete equivalence of the classical and quantum-mechanical statistical descriptions, as far as they depend on this region. The way to prove this is to show that the usual basical assumptions of classical and quantum-mechanical statistical mechanics are equivalent. These basical assumptions are the equal $a$-priori 
probabilities of all points in the $(\boldsymbol{r}, \boldsymbol{p})$-phase space on the one hand and of all eigenstates on the other hand.

An elementary proof of this equivalence follows from the Bohr-Sommerfeld quantization rule

$$
\iint_{H(p, q) \leq E} \mathrm{~d} p \mathrm{~d} q=\left(n+\frac{1}{2}\right) h
$$

which prescribes in the one-dimensional case a surface $h$ in $(p, q)$-space to each eigenstate. However, we would like to see this equivalence more explicitly in terms of localized wave-packets, which correspond directly to the classical particles. For this purpose we start from a set of $N$ neighbouring eigenstates in the quasi-classical region. A unitary transformation can be applied on this set, resulting in $N$ localized packets all moving classically in their WKB-approximation. The crucial point is now that at any given moment these localized particles are uniformly distributed over that part of phase space that corresponds to the energy shell of the used eigenstates, thus corresponding to the classical distribution.

In the first section, this procedure is worked out in detail for an arbitrary one-dimensional problem. In the second section, this is generalized for a three-dimensional spherical potential, in which we are especially interested in view of applications to the hydrogen plasma.

The density of the points of localization of the transformed set can be interpreted as a probability function. Such functions are considered in the third section. From this point of view, a new light is thrown on the classical divergence of the pair-correlation function of oppositely charged particles in a plasma.

Finally, in the last section, we give a direct proof of the equivalence of quantum and classical statistics by calculating the expectation value of an arbitrary observable first as an integral over phase space, then as a trace over the eigenstates. In the WKB-approximation both methods give the same result.

$\S 1$. Orthogonal wave packets in one dimension. Let us consider some onedimensional potential function $V(x)$ and an eigenfunction belonging to a discrete energy value $E$. We assume that there are exactly two points $a$ and $b$ with $a<b$ for which $V(x)=E$; these are the classical turning points. This can always be generalized to continuous states by enclosing the system in a large box, in which case the turning points lie on the walls of the box.

The WKB-approximation of the eigenfunction can be written as

$$
\psi_{E}(x) \sim \frac{1}{\sqrt{p(x)}}\left\{\cos \frac{1}{\hbar} \int_{x}^{b} \mathrm{~d} y p(y)-\frac{\pi}{4}\right\}
$$


where $p(x)$ is the local momentum

$$
p(x)=\sqrt{2 \mu[E-V(x)]}
$$

and $\mu$ the mass of the particle. The possible eigenvalues are given by the quantization rule

$$
\int_{a}^{b} \mathrm{~d} y p(y)=\hbar\left(n+\frac{1}{2}\right) \pi
$$

Each energy is thus characterized by a number $n$, denoting the number of nodes of the wave function between the turning points. We consequently indicate the energies with $E_{n}$, the eigenfunctions with $\psi_{n}$ and the momenta with $p_{n}(x)$.

As pointed out in the introduction, we suppose that we have a region in the energy spectrum where the density of levels is very high, which enables us to construct classically behaving wave packets. We now look for an explicit condition on this density. We need a large number of wave functions, approximately corresponding to the same values for all classically measurable quantities. For instance, it will be very important for our results that the turning points do not appreciably vary over the wave functions used in the packet. We can express this more quantitatively by the condition that the distances $\Delta a$ and $\Delta b$ between the turning points of two neighbouring levels must be much smaller than some characteristic length as e.g. $(b-a)$ :

$$
\Delta a \approx\left|\left(\frac{\mathrm{d} V}{\mathrm{~d} x}\right)^{-1}\right|_{x=a} \Delta E \ll b-a,
$$

which leads to

and equally

$$
\Delta E \ll(b-a)\left|\frac{\mathrm{d} V}{\mathrm{~d} x}\right|_{x=a}
$$

$$
\Delta E \ll(b-a)\left|\frac{\mathrm{d} V}{\mathrm{~d} x}\right|_{x=b}
$$

We defined $\Delta E$ as the spacing in energy between two neighbouring levels. It is approximately given by

$$
\Delta E=\frac{\hbar \pi / \mu}{\int_{a}^{b} \mathrm{~d} x p^{-1}(x)} .
$$

To prove this, let us express the quantization rule (3) for $(n+1)$, neglecting the difference of the turning points.

$$
\frac{\hbar \pi}{\sqrt{2 \mu}}\left(n+1+\frac{1}{2}\right)=\int_{a}^{b} \mathrm{~d} x \sqrt{E_{n}+\Delta E-V(x)}
$$




$$
\approx \int_{a}^{b} \mathrm{~d} x \sqrt{E_{n}-V(x)}+\frac{\Delta E}{2} \int_{a}^{b} \frac{\mathrm{d} x}{\sqrt{E_{n}-V(x)}}+\ldots
$$

The first integral cancels against part of the left hand side and equation (5) follows immediately.

Now, we will construct some linear combinations of these eigenfunctions, that are localized and move according to classical laws. We will demonstrate that it is possible to choose between all these combinations $N$ orthogonal ones, and that the points of localization of this orthogonal set are distributed in the phase space according to the classical statistical rule.

Consider the linear combination

$$
\Psi^{+}\left(x ; x_{0}\right)=\sum_{n=0}^{N-1} \psi_{n^{0}+n}(x) \exp \left\{+\frac{i}{\hbar} \int_{x_{0}}^{b} \mathrm{~d} y p_{n^{0}+n}(y)-\frac{i \pi}{4}\right\} .
$$

To show that this represents a localized wave, we substitute the WKBapproximation (1) and use the Taylor expansion

$$
p_{n^{0}+n}(x)=p_{n^{0}}(x)+\frac{n \mu}{p_{n^{0}}(x)} \Delta E+\ldots
$$

where also $\Delta E$ may be calculated with $p_{n^{\circ}}$, the error being of the same order of magnitude as the neglected third term in this expansion. We obtain

$$
\begin{aligned}
\Psi^{+}\left(x ; x_{0}\right) \sim \frac{1}{2 \sqrt{p_{n^{0}}(x)}}\left[\exp \left\{\frac{i}{\hbar} \int_{x_{0}}^{x} \mathrm{~d} y p_{n^{0}}(y)\right\} \cdot \sum_{n=0}^{N-1} \exp \left\{\mathrm{i} \pi n \frac{\int_{x_{0}}^{x} \mathrm{~d} y p_{n^{0}}^{-1}(y)}{\int_{a}^{b} \mathrm{~d} y p_{n^{0}}^{-1}(y)}\right\}\right. \\
-i \exp \left\{\frac{i}{\hbar} \int_{x}^{b} \mathrm{~d} y p_{n^{0}}(y)+\frac{i}{\hbar} \int_{x_{0}}^{b} \mathrm{~d} y p_{n^{0}}(y)\right\} \\
\left.\sum_{n=0}^{N-1} \exp \left\{i \pi n \frac{\int_{x}^{b} \mathrm{~d} y p_{n^{0}}^{-1}(y)+\int_{x_{0}}^{b} \mathrm{~d} y p_{n^{0}}^{-1}(y)}{\int_{a}^{b} \mathrm{~d} y p_{n^{0}}^{-1}(y)}\right\}\right]
\end{aligned}
$$

If $x_{0}$ satisfies $a<x_{0}<b$, the second term is negligibly small, whereas the first one is significant only in the neighbourhood of $x=x_{0}$. This can be seen from the behaviour of the function

$$
F_{N}(\alpha)=\sum_{n=0}^{N-1} \exp (i \pi n \alpha)
$$

which has peaks of height $N$ when $\alpha$ equals zero or an even number, and 
which oscillates rapidly with small amplitude between these values. The wave packet $\Psi^{+}\left(x ; x_{0}\right)$ is thus localized at $x_{0}$.

The classical movement of the point of localization is a well known fact, and can easily be demonstrated by multiplying the wave functions $\psi_{n^{0}+n}$ with their time-dependent factor

$$
\exp \left\{-\frac{i t E_{n^{0}+n}}{\hbar}\right\} \approx \exp \left\{-\frac{i t E_{n^{0}}}{\hbar}\right\} \exp \left\{-i \pi n \frac{t / \mu}{\int_{a}^{b} \mathrm{~d} y p_{n^{0}}^{-1}(y)}\right\} .
$$

One verifies easily that the movement goes in the positive $\mathrm{x}$ direction, which was anticipated by the plus sign attached to $\Psi$. When the point of localization arrives at the turning point $b$, the first and second term in the expression (8) change their role: the first becomes negligible whereas the second one now represents the returned particle moving in the negative direction. We could have started with a packet moving in the negative direction, if we had made the combination

$$
\Psi-\left(x ; x_{0}\right)=\sum_{n=0}^{N-1} \psi_{n^{0}+n}(x) \exp \left\{-\frac{i}{\hbar} \int_{x_{0}}^{b} \mathrm{~d} y p_{n^{0}+n}(y)+\frac{i \pi}{4}\right\} .
$$

Our main purpose was to show that we could make an orthogonal transformation into localized packets. Thus far we only looked at the possible linear combinations we could make out of the set of $N$ eigenfunctions with which we started. We still have to show that indeed $N$ of these combinations are mutually orthogonal. If $N$ is an even number, we can always find $N / 2$ points $x_{k}$ such that

$$
\int_{a}^{x_{k}} \mathrm{~d} y p_{n^{0}}^{-1}(y)=\frac{2 k-1}{N}-\int_{a}^{b} \mathrm{~d} y p_{n^{0}}^{-1}(y)
$$

with $k=1,2, \ldots N / 2$. The interpretation of this condition is that a classical particle spends equal times between each pair of neighbouring points $x_{k}$. We then localize both $a \Psi^{-}$and a $\Psi^{+}$. about each $x_{k}$. This set of $N$ wave packets is a linear transformation of the $N$ eigenfunctions $\psi_{n^{0}+n}$. Besides, they are all mutually orthogonal, as can be shown, using the orthogonality of the initial eigenfunctions. For instance

$$
\begin{aligned}
\int \mathrm{d} x\left[\Psi^{+}\left(x ; x_{k}\right)\right]^{*} \Psi^{+}\left(x ; x_{j}\right) & \sim \sum_{n=0}^{N-1} \exp \left\{\frac{i}{h} \int_{x_{j}}^{x_{k}} \mathrm{~d} y p_{n^{0}+n}(y)\right\} \sim \\
& \sim \sum_{n=0}^{N-1} \exp \left\{i \pi n \frac{\int_{x_{j}}^{x_{k}} \mathrm{~d} y p_{n^{0}}^{-1}(y)}{\int_{a}^{b} \mathrm{~d} y p_{n^{0}}^{-1}(y)}\right\}=\sum_{n=0}^{N-1} \exp \left[\frac{2 \pi i n}{N}(k-j)\right]=N \delta_{j k} .
\end{aligned}
$$




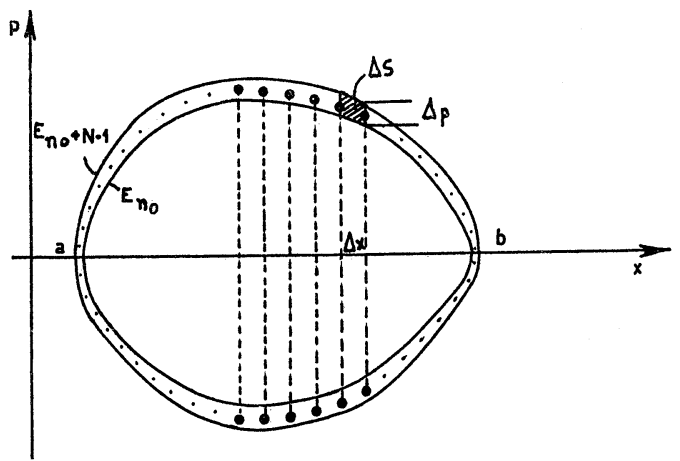

Fig. 1

The orthogonality can also be shown between each $\Psi^{+}$and $\Psi^{-}$. Finally, we remark that the procedure can be slightly modified in the case of odd $N$. This can be done, e.g., by choosing different sets $\left\{x_{k}\right\}$ for the $\Psi^{+}$and the $\Psi^{-}$.

Our last task is to demonstrate the equivalence between classical and quantum statistics. We therefore draw in the two-dimensional phase space $(x, p)$ the curves of constant energy for $E_{n^{0}}$ and $E_{n^{0}+N-1}$ (figure 1). They enclose a narrow ring in which the $N$ localized packets move around. At a given moment the points of localization will occupy the sites $\left[x_{k}, \pm p\left(x_{k}\right)\right]$. These $N$ points have equal probabilities to be occupied, because the wave functions from which we started had equal probabilities and the linear combinations were constructed with simple phase factors. Furthermore, there are no other independent localized waves possible in the same ring.

This means that quantum mechanics allows one and only one particle to be situated in a surface element in phase space, given by

$$
\Delta S=\Delta x \cdot \Delta p(x) \text {. }
$$

Classical statistical mechanics requires that the surfaces of all these phase space elements are equal, for they have equal occupation probabilities. Now, from (12)

$$
\Delta x \approx \frac{2 p_{n^{0}}(x)}{N} \int_{a}^{b} \mathrm{~d} y p_{n^{0}}^{-1}(y)
$$

and from the definition (2) of $p(x)$

$$
\Delta p(x) \approx \frac{\mu}{p_{n^{0}}(x)} N \Delta E=\frac{N \hbar \pi}{p_{n^{0}}(x) \int_{a .}^{b} \mathrm{~d} y p_{n^{0}}^{-1}(y)} .
$$

This gives, in agreement with the uncertainty relations,

$$
\Delta S=h
$$

which is constant over the whole phase space. 
§2. Orthogonal wave packets for the central-force problem. We will now extend the results of the previous section to a three-dimensional spherically symmetric potential. It is well known that the eigenfunctions are characterized by three quantum numbers $n, l$ and $m$, and can be separated as

$$
\psi_{n, l, m}(r, \theta, \phi)=Y_{l}^{m}(\theta, \phi) R_{n, l}(r)
$$

with energies $E_{n, l} \cdot Y_{l}^{m}$ is the usual spherical harmonic function. The radial eigenfunction can be written in the WKB-approximation as

$$
R_{n, l}(r) \sim \frac{1}{r \sqrt{p(r)}} \cos \left\{\frac{1}{\hbar} \int_{r}^{b} \operatorname{dy} p(y)-\frac{\pi}{4}\right\} .
$$

$p(r)$ is the radial momentum, given by

$$
p_{n, l}(r)=\sqrt{2 \mu\left[E_{n, l}-V(r)-\frac{\hbar^{2} l^{2}}{2 \mu r^{2}}\right]}
$$

and $b$ is the upper turning point, here defined by $p(b)=0$.

As in the previous section, we would like to construct a wave packet out of some functions that have approximately the same energy $E_{0}$ and the same quantum numbers $l_{0}$ and $m_{0}$. We again neglect possible differences in the turning points.

The spacing in the energy levels is calculated from (5) and approximately given by

$$
\Delta E=E_{n+1, l}-E_{n, l} \approx \frac{\hbar \pi / \mu}{\int_{a}^{b} \mathrm{~d} r p_{n_{0}, l_{0}}^{-1}(r)} .
$$

We thus neglect the $n$ - and $l$-dependence of this $\Delta E$. It follows that it must be possible to find for all $l$ a number $\boldsymbol{v}(l)$ such that

$$
\left|E_{n_{0}, l_{0}}-E_{n_{0}+\nu(l), l}\right|=|\varepsilon(l)| \leq \frac{\Delta E}{2} .
$$

We now relabel the energy values by changing the indices $(n, l)$ into $\left(n^{\prime}, l\right)$ with $n^{\prime}=n+v(l)$. Obviously $v\left(l_{0}\right)=0$. The energies $E_{n^{\prime}, l}$ are now approximately independent of $l$. Indeed:

$$
\begin{aligned}
E_{n^{\prime}, l_{0}}- & E_{n^{\prime}, l}=E_{n, l_{0}}-E_{n+v(l), l}= \\
& =\left[E_{n_{0}, l_{0}}+\left(n-n_{0}\right) \Delta E\right]-\left[E_{n_{0}+v(l), l}+\left(n-n_{0}\right) \Delta E\right]=\varepsilon(l)
\end{aligned}
$$

which can be neglected. It is well known that for the Coulomb potential, in which we are especially interested, $\varepsilon(l)$ exactly vanishes.

From now on, we drop the accent of $n^{\prime}$ and $n$ will represent the new label. 
A wave-packet out of $\left(N_{n} N_{l} N_{m}\right)$ eigenfunctions (17) can be written as

$$
\Psi(r, \theta, \phi)=\sum_{j=0}^{N_{n}-1} \sum_{k=0}^{N_{l}-1} a(j, k) R_{n_{0}+j, l_{0}+k}(r) \sum_{s=0}^{N_{m}-1} b(s) Y_{l_{0}+k}^{m_{0}+s}(\theta, \phi) .
$$

Our first step will be to show that we can choose the $b(s)$ such that the wave function is localized in a single plane through the centre, which is the first requirement for a classical central-force movement. We therefore need an asymptotic approximation to the spherical harmonic function for large $(l, m)$-values. Such an approximation was given by Jeffreys and J effreys $\left.{ }^{2}\right)$ for the associated Legendre-function $P_{l}^{m}$, connected with the spherical harmonic function by the relation

$$
Y_{l}^{m}(\theta \phi)=C \mathrm{e}^{i m \phi} P_{l}^{m}(\cos \theta),
$$

where $C$ is a normalization constant. Their result is obtained by a method of steepest descent, and in an appendix we show that one can obtain the same formulas with a WKB-procedure. The resulting approximation is given, with an other constant $C^{\prime}$, by

$$
P_{l}^{m}(\cos \theta)=C^{\prime} \frac{\sin \left[A(l, m ; \theta)-m B(l, m ; \theta)+\frac{\pi}{4}\right]}{\sqrt[4]{l^{2} \sin ^{2} \theta-m^{2}}}
$$

with

$$
A(l, m ; \theta)=\arccos \frac{l \cos \theta}{\sqrt{l^{2}-m^{2}}}
$$

and

$$
B(l, m ; \theta)=\arccos \frac{m \operatorname{cotg} \theta}{\sqrt{l^{2}-m^{2}}}
$$

This result is valid only between the turning points, which means

$$
l^{2} \sin ^{2} \theta-m^{2}>0 ;
$$

for other $\theta$-values the function practically vanishes.

For constructing the wave-packet (21) we now choose

$$
b(s)=\exp \left\{\text { is } B\left(l, m_{0} ; \theta_{0}\right)\right\}
$$

where $\theta_{0}$ must lie in the range of values satisfying (25). To show that with this choice the wave is localized in a plane, we have to consider separately the two exponential terms of the sine-function of the approximation (23). The separation into these terms is expressed by the obvious notation

$$
Y_{l}^{m}(\theta, \phi)=\frac{1}{2 i}\left[{ }^{(+)} Y_{l}^{m}(\theta, \phi)-{ }^{(-)} Y_{l}^{m}(\theta, \dot{\phi})\right]
$$

and we call these terms respectively the positive and negative frequency 
parts. With a first-order Taylor approximation in $s / m_{0}$, we then have

$$
\sum_{s=0}^{N_{m}-1} b(s)^{( \pm)} Y_{l}^{m_{0}+s}(\theta, \phi)={ }^{( \pm)} Y_{l}^{m_{0}}(\theta, \phi) \frac{\mathrm{e}^{i \alpha N_{m}}-1}{\mathrm{e}^{i \alpha}-1}
$$

where

$$
\alpha=\phi \mp B\left(l, m_{0} ; \theta\right)+B\left(l, m_{0} ; \theta_{0}\right) .
$$

The function (28) is localized about $\alpha$ equal to zero or a multiple of $2 \pi$. The $B$-functions can only attain values within a range of length $\pi$, which means that the two terms in the separation (27) give rise to localization about two values $\phi(+; \theta)$ and $\phi(-; \theta)$, covering together the whole $\phi$-interval when $\theta$ runs over its permitted range. This relation between the $\theta$ - and $\phi$-values describes a plane through the origin, and it can easily be verified that the spherical angles of the axis normal to this plane are given by

$$
\begin{gathered}
\theta^{\prime}=\operatorname{arc} \cos m_{0} / l \\
\phi^{\prime}=\pi-B\left(l, m_{0} ; \theta_{0}\right) .
\end{gathered}
$$

The value of $\theta^{\prime}$ was to be expected from the physical meaning of the $m$ index.

Before proceeding with the investigation of the different wave packets in this plane, we need a transcription of the localized wave (28). As there is a functional relation between the $\phi$ - and $\theta$-coordinates of all points in the plane of localization, the harmonic function can only depend on a single coordinate, for instance the polar angle $\xi$ with respect to the intersection of this plane with the $x z$ plane. A transformation from $(\theta, \phi)$ to this $\xi$ yields for all points in the plane

$$
{ }^{( \pm)} Y_{l}^{m_{0}}(\theta, \phi) \sim \mathrm{e}^{i l \xi} .
$$

This is in agreement with the possibility of choosing the $z$ axis perpendicular to the plane of localization, in which case

$$
Y_{l}^{l}(\theta, \phi) \sim \mathrm{e}^{i l \phi} \sin ^{l} \theta ;
$$

the first factor is of the form (31), and the second factor localizes the wave in the $x y$ plane. In the following, we suppose that we have made this particular choice of $z$ axis. We now construct the different localized wave packets in the $x y$ plane. The WKB-approximation to the radial function $R_{n, l}$ again splits up in positive and negative frequency parts ${ }^{( \pm)} R_{n, l}$. Let us choose for the factors $a(j, k)$ in the packet (21) the following functions:

$$
a^{ \pm}\left(j, k ; r_{0}, \phi_{0}\right)=\exp \left\{ \pm \frac{i}{\hbar} \int_{r_{0}}^{b} \mathrm{~d} y p_{n_{0}+j, l_{0}+k}(y)-i\left(l_{0}+k\right) \phi_{0}\right\} .
$$

With these functions, we can build two different wave packets $\Psi^{+}$and $\Psi^{-}$. 
Each of them has a positive and a negative frequency part from the splitting up of $R_{n, l}$, indicated as before with a sign between brackets. The negative frequency part of the packet constructed with the $a^{+}$will then be proportional to

$$
\begin{aligned}
(-) \Psi^{+}\left(r, \phi, t ; r_{0}, \phi_{0}\right) \sim \sum_{j=0}^{N_{n}-1} \sum_{k=0}^{N_{l}-1} \exp \left\{\frac{i}{\hbar} \int_{r_{0}}^{r} \mathrm{~d} y p_{n_{0}+j, l_{0}+k}(y)+\right. \\
\left.+i\left(l_{0}+k\right)\left(\phi-\phi_{0}\right)-\frac{i t}{\hbar} E_{n_{0}+j}\right\},
\end{aligned}
$$

where we included the time dependence. With a first order Taylor expansion in $j / n_{0}$ and $k / l_{0}$, this gives

$$
(-) \Psi^{+} \sim \sum_{i} \mathrm{e}^{i j \gamma} \sum_{k} \mathrm{e}^{i k \zeta}
$$

with

$$
\gamma=\frac{\Delta E}{\hbar}\left[\int_{r_{0}}^{r} \frac{\mathrm{d} y}{p_{n_{0}, l_{0}}(y)}-t\right]
$$

and

$$
\zeta=\phi-\phi_{0}-\hbar l_{0} \int_{r_{0}}^{r} \frac{\mathrm{d} y}{y^{2} p_{n_{0}, l_{0}}(y)} .
$$

The packet is localized around $\gamma$ and $\zeta$ equal to zero (or a multiple of $2 \pi$ ). For time zero, the negative frequency part $(-) \Psi^{+}$of the packet is thus localized at $\left(r_{0}, \phi_{0}\right)$. A similar calculation shows that the positive frequency part of the same packet almost vanishes for time zero.

The classical movement of the wave packet is described by the equations $\gamma=\zeta=0$, which are well known equations in classical mechanics ${ }^{3}$ ). Analogous to the one-dimensional case, the positive and negative frequency parts of the wave packet change their roles when the particle arrives at a turning point. The only difference lies in the fact that the particle, although returning in the $r$-coordinate, moves steadily ahead in the $\phi$-coordinate.

The localized linear combinations that we constructed from a set of $\left(N_{n} N_{l} N_{m}\right)$ neighbouring eigenfunctions depend on three parameters: $\theta_{0}, r_{0}$ and $\phi_{0}$, and there are two combinations for each set: $\Psi^{+}$and $\Psi^{-}$. As in the one-dimensional case, we now indicate how to obtain $\left(N_{n} N_{l} N_{m}\right)$ mutually orthogonal combinations. We first choose $N_{m}$ values $\theta_{0_{s}}$ for $\theta_{0}$, such that the values for the $\phi_{s}^{\prime}$ corresponding to them by the relation (30b) satisfy

$$
\phi_{s}^{\prime}=\frac{2 \pi s}{N_{m}} \quad \text { and } \quad s=1,2, \ldots, N_{m}
$$


for $\phi_{0}$. we take the values

$$
\phi_{0 k}=\frac{2 \pi k}{N_{l}} \quad \text { and } \quad k=1,2, \ldots, N_{l}
$$

and for $r_{0}$ the $N_{n} / 2$ values defined by

$$
\int_{a}^{r_{0 j}} \mathrm{~d} y p_{n_{0}, l_{0}}^{-1}(y)=\frac{2 j-1}{N_{n}} \int_{a}^{b} \mathrm{~d} y p_{n_{0}}^{-1}, l_{0}(y)=\frac{\hbar \pi}{\mu} \frac{2 j-1}{N_{n} \Delta E} .
$$

The $\left(N_{n} N_{l} N_{m}\right)$ wave packets $\Psi^{ \pm}\left(r, \theta, \phi, t ; r_{0_{j}}, \theta_{0_{s}}, \phi_{0_{k}}\right)$ then form an orthogonal set, as is easily verified.

To clarify the interpretation of this set, we remark that a different $\phi_{0}$ corresponds to a different orientation of some characteristic axis of the orbit, as e.g. the major axis of an elliptical orbit. A different $\boldsymbol{r}_{0}$-value corresponds to a different starting point at $t=0$ on the same orbit. Whereas the different $\phi_{0}$-values have an equal spacing between them, the different $r_{0}$-values are such that a classical particle spends equal times between them. This time spacing is exactly one $N_{n}$-th part of an up-and-down movement in the radial coordinate. For the general central force, the particle will not necessarily regain its original $\phi$-value after such an up-and-down movement, so that there is no real periodicity. A $\Psi^{+}$corresponds to a particle that is at $t=0$ in a state of increasing radial distance whereas a $\Psi^{-}$corresponds to a decreasing radial distance.

We have thus verified that a set of quantum-mechanical wave functions with large quantum numbers can be changed by an orthogonal transformation into a set of classically moving localized packets. When the original set has a narrow spread about some mean value for the observable quantities as energy and angular momentum, the transformed set consists of orbits, which are identical except for a uniform distribution of the directions of the normal and semi-major axis, and except for "equal time distances" between the localized points on the same orbit.

The demonstration of the equivalence between classical and quantummechanical statistics can be performed along almost the same lines as the demonstration at the end of the previous section. We omit here this proof, because the same equivalence will follow directly from the probability distribution functions derived in the next section.

§3. Probabilities and the correlation function. In this section we derive some probability functions. They are approximations to the quantummechanical expectation values, valid in the semi-classical region where the WKB-methods are applicable. It will be seen in the next section that the results are classically predicted.

To start with, let us ask for the probability $g(E, l \mid r)$ for finding a particle 
at a distance $r$ from the centre of force, if it is known to have an energy $E$ and an angular momentum quantum number $l$. Quantum-mechanically, this is given by the square modulus of the wave function:

$$
g(E, l \mid \gamma)=\left|\Psi_{E, l}\right|^{2}
$$

which is normalized to unity for a volume integration,

$$
\int \mathrm{d} \boldsymbol{r} g(E, l \mid r)=1 .
$$

In classical statistical mechanics, probability functions are calculated as averages over an ensemble, with the basical assumption of equal probabilities for equal volume elements in phase space. To convert the expression (40) into a useful form we shall also take an ensemble, consisting of a discrete set of neighbouring wave functions in the semi-classical region of the spectrum. The only assumption is that all eigenfunctions of this set have equal probabilities. In the previous section we showed that a set of $N_{n}$ eigenfunctions on a given orbit is equivalent to $N_{n}$ localized particles at different radial distances. The assumption for equal probabilities for the eigenfunctions transforms now to equal probabilities for the different localized particles. This means that the probability function $g(E, l \mid r)$ will be inversely proportional to the distance $\Delta(r)$ between two neighbouring localized particles. As our ensemble contains also a packet of $l$-values and all allowed $m$-values, there will be complete spherical symmetry; the probability function will then be inversely proportional to the volume element $4 \pi r^{2} \Delta(r)$ between two allowed neighbouring $r$-values. Imposing the normalization (41) we must have

$$
g(E, l \mid r)=\frac{2}{4 \pi r^{2} \Delta(r) N_{n}} .
$$

$\Delta(r)$ can be calculated from (39), with which we get

$$
g(E, l \mid r)=\frac{\mu \Delta E}{2 \pi r^{2} h p(r)} \Theta\left[p^{2}(r)\right]
$$

where $p(r)$ is the radial momentum (19) and $\Theta$ the unit step function defined by

$$
\begin{array}{rlll}
\Theta(x)=1 & \text { if } & x>0 \\
=0 & \text { if } & x<0 .
\end{array}
$$

This step functon arises from the condition that $r$ must lie between the turning points.

A useful extension of this conditional probability function is the correlated $a$-priori probability function $g(E, l, v)$ for finding the particle simultaneously with energy $E$, angular momentum quantum number $l$ and radial coordinate $r$. It is defined by

$$
g(E, l, r)=\rho(E) \rho(l) g(E, l \mid r)
$$


where $\rho(E)=1 / \Delta E$ is the density of the energy spectrum and $\rho(l)=$ $=2 l+1 \approx 2 l$ the degeneracy of the angular momentum. The result is

$$
g(E, l, r)=\frac{\mu l}{\pi r^{2} h p(r)} \Theta\left[p^{2}(r)\right] .
$$

The spacing $\Delta E$ disappeared, and this result can be generalized to free states, after the spherical enclosure is allowed to tend to infinity.

We are now able to write down an expression for the total probability for finding a particle at a distance $r$ from the centre of force. By doing this, we have in mind the pair correlation function for particles in a plasma. The long distance behaviour of this function is very well investigated nowadays, but short distances still offer some open questions for oppositely charged ions. For such distances, we forget the rest of the plasma, considering only the two ions of interest in their centre-of-mass system. We assume that the occupation probability of a state with energy $E$ is given by $\exp (-\beta E)$. Some states will lie in a region of the spectrum where our transformation into localized particles is allowed; they will give a contribution of the form (46), which we now consider as a continuous function of $E$ and $l$. Other eigenstates do not allow such a treatment and have to be considered separately. This gives for the pair correlation function $g(r)$ :

$$
Z g(r)=\underset{E, l, m}{\sum^{0}} \mathrm{e}^{-\beta E}\left|\Psi_{E, l, m}(r)\right|^{2}+\int^{0} \mathrm{~d} E \int^{0} \mathrm{~d} l \mathrm{e}^{-\beta E} g(E, l, r) .
$$

$Z$ is the partition function, normalizing the Boltzmann weight-factors. The domains of the summation and integration have to be chosen complementary, covering together all allowed values; this is indicated with the 0 . This expression, derived from quantum-mechanics and WKB-methods, reproduces the old classical result if the integral is extended over all possible values. To demonstrate this, we first carry out the $l$-integration, defining a new probability function

$$
g(E, r)=\int_{0}^{\infty} \mathrm{d} l g(E, l, r)=\frac{2 \pi(2 \mu)^{\frac{3}{2}}}{h^{3}} \sqrt{E-V(r)} \Theta[E-V(r) .]
$$

The classical pair-correlation function $g_{C}$ becomes

$$
Z g_{C}(r)=\int_{-\infty}^{+\infty} \mathrm{d} E \mathrm{e}^{-\beta E} g(E, r)=\left(\frac{2 \pi \mu}{\beta h^{2}}\right)^{\frac{3}{2}} \mathrm{e}^{-\beta V(r)},
$$

and this diverges when $V(r)=-\infty$, which occurs at the origin of an attractive Coulomb potential.

The combined expression (47) has the advantage that the quantum effects that remove the divergence are contained in a finite number of terms, 
whereas the rest has the form of an elementary integral. The number of quantum terms has to be chosen according to the desired accuracy. It will also depend on the temperature, because lower temperatures give greater weight factors to the quantum states.

To see how the divergence is removed, let us calculate the integral in (47) with some lower limit $E_{0}$ for the energies, which may be negative. If we neglect the non-classical behaviour of the small number of $s$-states $(l=0)$, we can use the function $g(E, r)$. We have

$$
Z g(r)=\sum^{E_{0}} \mathrm{e}^{-\beta E}\left|\Psi_{E}(r)\right|^{2}+\int_{E_{0}}^{\infty} \mathrm{d} E \mathrm{e}^{-\beta E} g(E, r) .
$$

The integral can be written as

$$
\int_{E_{0}}^{\infty} \mathrm{d} E \mathrm{e}^{-\beta E} g(E, r)=\left(\frac{2 \pi \mu}{\beta h^{2}}\right)^{\frac{3}{2}} \mathrm{e}^{-\beta V(r)} \Xi\left[E_{0}-V(r)\right]
$$

The function $\Xi$ turns out to be given by

$$
\Xi(y)=1+\Theta(y)\left[2 \sqrt{\frac{y}{\pi}} \mathrm{e}^{-y}-\Phi(\sqrt{ } y)\right]
$$

where $\Theta$ is again the unit step function (44) and $\Phi$ is the usual error function. The exponential divergence has disappeared. There remains a small divergence from $\sqrt{ } y$, without physical consequences: for a Coulomb potential this would be proportional to $r^{-\frac{1}{2}}$, which drops out by integration.

The main quantum effect for the convergence of the correlation function thus seems to be the existence of some lower limit for the allowed energy values. Attempts have been made for obtaining a convergent pair correlation function by $\mathrm{Lamb}^{4}$ ) and by Trubnikov and Elesin ${ }^{5}$ ), without paying attention to this main quantum effect. The former uses an asymptotic approximation in $h$, and the question can be raised whether a further approximation would not reveal new divergent factors. The latter authors assume complete ionization and suggest that their convergence results from the use of quantum wave functions. Complete ionization, however, means positive energies; we showed that even with classical expressions such a cutoff in the energies is sufficient for a convergent result.

$\S 4$. Correspondence with classical statistics. The basical assumption of the classical statistical mechanics of a system of $N$ particles is that equal volume elements in the same energy shell of the $6 N$-dimensional phase space have equal occupation probabilities. When two particles come very close together, their potential energy will almost completely be determined by their relative positions. One can neglect the influence of all other particles and separate out the center-of-mass motion of this pair. The statistical assumption reduces 
in the 6-dimensional phase space of their relative coordinates to the distribution probability of a canonical ensemble, again with equal probabilities for equal volume elements with the same energy. The connection with quantum mechanics is usually expressed by saying that the Heisenberg uncertainty relations impose the condition that a phase-space volume of $h^{3}$ corresponds to one quantum state. This can be written symbolically as

$$
\sum_{E} \Leftrightarrow \int \frac{\mathrm{d} \boldsymbol{r} \mathrm{d} \boldsymbol{p}}{h^{3}}
$$

The easiest way of showing that these assumptions are consistent with our previous results is by observing that the classical part of (53) predicts a density of states $h^{-3}$ in $(\boldsymbol{r}, \boldsymbol{p})$-space. This is transformed into $(\boldsymbol{r}, E)$-space by

$$
\mathrm{d} \boldsymbol{p}=2 \pi(2 \mu)^{\frac{3}{2}} \sqrt{E-V(r)} \mathrm{d} E
$$

and with the factor $h^{-3}$ we obtain exactly the density function $g(E, r)$, given by (48).

As remarked at the end of the second section, it can directly be verified that the equivalence (53) is true in the semi-classical region by showing that the points of localization of the constructed wave-packets lie at the corners of 6-dimensional parallelepipeds of volume $h^{3}$. However, we will leave here our wave-packets and prove the equivalence between classical and quantum statistics by calculating directly the expectation value of an arbitrary function. We calculate this first classically, then quantum-mechanically in the WKB-approximation, and obtain the same result.

Let $F\left(r, E, L, L_{z}\right)$ be any function of radial coordinate, energy, angular momentum and its $z$-component of a particle with mass $\mu$ in a spherical potential $V(r)$. It is evident that a Boltzmann probability factor can be built in. The classical expectation value is given as

$$
\langle F\rangle_{C}=h^{-3} \int \mathrm{d} \boldsymbol{r} \int \mathrm{d} \boldsymbol{p} F\left(r, E, L, L_{\boldsymbol{Z}}\right) .
$$

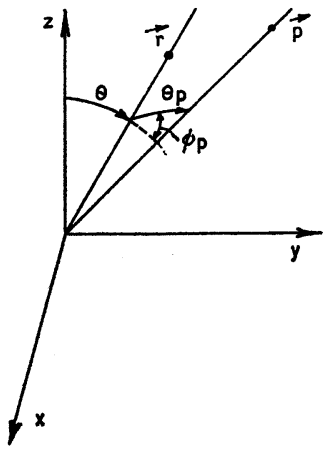

Fig. 2

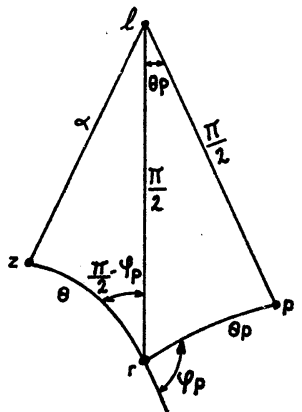

Fig. 3 
We now make a transformation from $\boldsymbol{p}$ to the variables $E, L$ and $L_{\boldsymbol{Z}}$. Let $\boldsymbol{r}$ be determined by the usual polar coordinates $(r, \theta, \phi)$ where $\theta$ is the angle between $\boldsymbol{r}$ and $z$ axis. We then determine $\boldsymbol{p}$ by the polar coordinates $\left(p, \theta_{p}\right.$, $\phi_{p}$ ) where $\boldsymbol{r}$ plays the role of the polar axis and $\phi_{p}$ is the angle between the planes $(\boldsymbol{z} \boldsymbol{r})$ and $(\boldsymbol{r} \boldsymbol{p})$. This is illustrated in fig. 2 , and repeated in fig. 3 , which is drawn on the surface of the unit sphere where $z, r, p, L$ respectively indicate the intersection points of the sphere with the $z$ axis, $\boldsymbol{r}, \boldsymbol{p}$ and the angular momentum $\boldsymbol{L}=\boldsymbol{r} \times \boldsymbol{p}, \alpha$ is the angle between $\boldsymbol{L}$ and $z$ axis. We then have

$$
\begin{aligned}
E & =\frac{p^{2}}{2 \mu}+V(r) \\
|L| & =p r \sin \theta_{p} \\
L_{Z} & =p r \sin \theta_{p} \cos \alpha=p r \sin \theta \sin \theta_{p} \sin \phi_{p} .
\end{aligned}
$$

The Jacobian of the transformation gives

$$
\mathrm{d} E \mathrm{~d} L \mathrm{~d} L_{Z}=\frac{r^{2} p^{3}}{\mu} \sin \theta \sin \theta_{p} \cos \theta_{p} \cos \phi_{p} \mathrm{~d} p \mathrm{~d} \theta_{p} \mathrm{~d} \phi_{p} .
$$

We further remark that the domain of $\left(\theta_{p}, \phi_{p}\right)$ covers four times the domain of $\left(L, L_{z}\right)$. Substituting the Jacobian and the limits of integration gives

$$
\begin{aligned}
\langle F\rangle_{C}=\frac{4 \pi \sqrt{2 \mu}}{h^{3}} \int_{0}^{\infty} \mathrm{d} r \int_{0}^{\infty} \mathrm{d} \theta \sin \theta \int_{V(r)}^{\infty} \mathrm{d} E \int_{0}^{r \sqrt{2 \mu[E-V(r)]}} \mathrm{d} L \int_{-L \sin \theta}^{L \sin \theta} \\
\frac{L F\left(r, E, L, L_{Z}\right)}{\sqrt{\left[E-V(r)-\frac{L^{2}}{2 \mu r^{2}}\right]\left(L^{2} \sin ^{2} \theta-L_{Z}^{2}\right)}}
\end{aligned}
$$

The quantum-mechanical expectation value of the same function has to be written as a trace, where $F$ is now a function of operators:

$$
\begin{aligned}
\langle F\rangle_{Q M}= & \operatorname{Tr} F\left(r, H, L, L_{Z}\right)= \\
& =\sum_{E} \sum_{l} \sum_{m} \int \mathrm{d} \boldsymbol{r}\left|\psi_{E, l, m}(r, \theta, \phi)\right|^{2} F(r, E, \hbar \sqrt{l(l+1)}, \hbar m) .
\end{aligned}
$$

We have written the trace with the wavefunctions (17), which are exact eigenfunctions of the operators $H, L$ and $L_{Z}$. We shall approximate these functions by WKB-methods, and in this same approximation we can replace $l(l+1)$ by $l^{2}$. The WKB-approximation of the eigenfunctions was already described in the previous sections, with formules (18) and (22)-(23). Their main characteristic is that they practically vanish outside some turning points, whereas they oscillate very rapidly in the physical region. It is only the square modulus of these functions that appears in our trace. We then 
suppose that the function $F$ is a smooth function in the sense that it does not change appreciably over the wavelength of the WKB-function. If this is true, we can forget about the oscillating factor in the WKB-approximations. We still have to determine the constants that appear in these functions, which is done by imposing the normalization of $|\psi|^{2}$, for instance by requiring that

$$
\int_{0}^{\infty} \mathrm{d} r r^{2}|R(r)|^{2}=1
$$

and

$$
\int_{0}^{2 \pi} \mathrm{d} \phi \int_{0}^{\pi} \mathrm{d} \theta \sin \theta|Y(\theta, \phi)|^{2}=1
$$

This determines the constants and we have

and

$$
\left|P_{n, l}^{(r)}\right|^{2} \approx \frac{\mu \Delta E}{\hbar \pi r^{2} p_{n, l}(r)}
$$

$$
\left|Y_{l}^{m}(\theta, \phi)\right|^{2} \approx \frac{l}{2 \pi^{2} \sqrt{l^{2} \sin ^{2} \theta-m^{2}}} .
$$

The normalization of $|R|^{2}$ is proved with relation (20). The last step in the transformation of the trace is the replacement of the summations over the eigenvalues by an integration. This introduces the density functions $\rho(l)=$ $=1, \rho(m)=1$ and $\rho(E)=1 / \Delta E$. The integration limits are determined from the WKB-turning points. We finally obtain

$$
\begin{aligned}
\langle F\rangle_{Q M}=\frac{\sqrt{2 \mu}}{\pi h} \int_{0}^{\infty} \mathrm{dr} \int_{0}^{\infty} \mathrm{d} \theta & \sin \theta \int_{V(r)}^{\infty} \mathrm{d} E \int_{0}^{(r / \hbar)} \sqrt{2 \mu[E-V(r)]} l \sin \theta \\
& \times \frac{l F(r, E, \hbar l, \hbar m)}{\sqrt{\left[E-V(r)-\frac{\hbar^{2} l^{2}}{2 \mu r^{2}}\right]\left(l^{2} \sin ^{2} \theta-m^{2}\right)}}
\end{aligned}
$$

The equivalence with the corresponding classical expression is evident.

This result is of course only valid if all eigenfunctions are suited for a WKBapproximation. In the general case one will need a combination of expressions (57) and (61). What part of the spectrum may be transformed into a classical calculation again depends on the desired accuracy and the temperature. Two kinds of errors are introduced: first the WKB-approximation together with the supposed continuity of the spectrum, and then the neglect of the squared oscillating factor in this approximation. The nature of the function $F$ itself will determine the gravity of these errors. As an example, if this function does not behave singularly for $L=0$, one can neglect for higher energies the non-classical behaviour of the low angular 
momentum states, because the number of such states is very small. This was done above for the calculation of the pair correlation function.

The next problem is the application of the outlined procedure to the calculation of some function defined for a system of particles, e.g. a plasma. If the relative coordinates of a close pair of particles can be separated out of this function, our method can be applied. From a certain distance on, however, the other particle coordinates will also be important. A careful investigation of the particular problem will in most cases permit the separation into a classical many-body region and a two-body region, partly classical partly quantum-mechanical. An example of such a procedure was worked out for the calculation of the partition function ${ }^{1}$ ), which will also be needed for the normalization of any other expectation value.

\section{APPENDIX}

We derive in this appendix a WKB-approximation for the associated Legendre-function $P_{l}^{m}$, which satisfies the differential equation

$$
\left(1-x^{2}\right) \frac{\mathrm{d}^{2} P(x)}{\mathrm{d} x^{2}}-2 x \frac{\mathrm{d} P(x)}{\mathrm{d} x}+\left[l(l+1)-\frac{m^{2}}{1-x^{2}}\right] P(x)=0 .
$$

Therefore, we try a solution of the form

$$
{ }^{( \pm)} P(x)=U(x) \mathrm{e}^{ \pm i W(x)}
$$

and get the set of equations

$$
\begin{gathered}
\left(1-x^{2}\right)\left(\frac{U^{\prime \prime}}{U}-W^{\prime 2}\right)-2 x \frac{U^{\prime}}{U}+\frac{l(l+1)\left(1-x^{2}\right)-m^{2}}{1-x^{2}}=0 \\
2 \frac{U^{\prime}}{U}+\frac{W^{\prime \prime}}{W^{\prime}}-\frac{2 x}{1-x^{2}}=0 .
\end{gathered}
$$

The last equation can be integrated to

$$
U(x)=U_{0} S^{-\frac{1}{2}}(x) ; W^{\prime}(x)=\frac{S(x)}{1-x^{2}},
$$

where $S(x)$ satisfies the equation

$$
\begin{aligned}
S^{2}=\left[l(l+1)\left(1-x^{2}\right)-m^{2}\right]+\left(1-x^{2}\right)^{2}\left[\frac{3}{4}\left(\frac{S^{\prime}}{S}\right)^{2}-\frac{S^{\prime \prime}}{2 S}\right]+ & \\
& +x\left(1-x^{2}\right) \frac{S^{\prime}}{S} .
\end{aligned}
$$

To obtain an approximate solution of this equation, asymptotically valid for large values of $l$ and $m$, we retain in the right hand side only the terms 
that are quadratic in those parameters, or

$$
S^{2} \approx l^{2}\left(1-x^{2}\right)-m^{2} .
$$

Approximate solutions of the initial differential equation are thus given by

$$
\text { ( }) P(\cos \theta)=U_{0} \frac{\exp \left\{ \pm i \int^{\theta} \mathrm{d} \theta \frac{\sqrt{l^{2} \sin ^{2} \theta-m^{2}}}{\sin \theta}\right\}}{\sqrt[4]{l^{2} \sin ^{2} \theta-m^{2}}}
$$

The indefinite integral can be evaluated as

$$
\int^{\theta} \mathrm{d} \theta \frac{\sqrt{l^{2} \sin ^{2} \theta-m^{2}}}{\sin \theta}=l A(l, m ; \theta)-m B(l, m ; \theta)
$$

where

$$
A(l, m ; \theta)=\int^{\theta} \mathrm{d} \theta \frac{l \sin \theta}{\sqrt{l^{2} \sin ^{2} \theta-m^{2}}}=\arccos \frac{l \cos \theta}{\sqrt{l^{2}-m^{2}}}
$$

and

$$
B(l, m ; \theta)=\int^{\theta} \mathrm{d} \theta \frac{m}{\sin \theta \sqrt{l^{2} \sin ^{2} \theta-m^{2}}}=\arccos \frac{m \operatorname{cotg} \theta}{\sqrt{l^{2}-m^{2}}}
$$

are the functions defined in the second section (24).

As is well known from the usual WKB-theory, only a special linear combination of the solutions (A.5) has an exponentially decaying behaviour outside the physical region. This physical solution was given in formula (23).

Acknowledgement. This work was performed during a stay at the University of Utrecht, Netherlands, and with the financial help of the Belgian “Nationaal Fonds voor Wetenschappelijk Onderzoek”. The author is indebted to Professor N. G. van Kampen and Dr. B. U. Felderhof for their critical discussions and valuable remarks.

Received 20-1-67

\section{REFERENCES}

1) Dekeyser, R., Physica 31 (1965) 1405.

2) Jeffreys, H, and Jeffreys, B. S., Methods of Mathematical Physics, Cambridge Univ. Press (1950), s. 658.

For a complete elaboration, cf. Robin, L., Fonctions Sphériques de Legendre et Fonctions Sphéroïdales, Gauthier-Villars, Paris (1958) II p. 256.

3) Goldstein, H., Classical Mechanics, Addison-Wesley (1950), equations (3.18) and (3.36).

4) Lamb, G. L., Phys. Rev. Letters 23 (1964) 683.

5) Trubnikov, E. A. and Elesin, V. F., Sov. Phys. J.E.T.P. 20 (1965) 866. 